TITLE:

\title{
Simulations of diocotron instability using a special-purpose computer, MDGRAPE-2
}

$\operatorname{AUTHOR}(\mathrm{S})$ :

Yatsuyanagi, Y; Kiwamoto, Y; Ebisuzaki, T; Hatori, T; Kato, $T$

CITATION:

Yatsuyanagi, Y ...[et al]. Simulations of diocotron instability using a special-purpose computer, MDGRAPE-2. PHYSICS OF PLASMAS 2003, 10(8): 3188-3195

\section{ISSUE DATE:}

2003-08

URL:

http://hdl.handle.net/2433/50245

\section{RIGHT:}

Copyright 2003 American Institute of Physics. This article may be downloaded for personal use only. Any other use requires prior permission of the author and the American Institute of Physics. 


\title{
Simulations of diocotron instability using a special-purpose computer, MDGRAPE-2
}

\author{
Yuichi Yatsuyanagi ${ }^{\mathrm{a})}$ and Yasuhito Kiwamoto \\ Graduate School of Human and Environmental Studies, Kyoto University, Sakyo, Kyoto 606-8501, Japan \\ Toshikazu Ebisuzaki \\ RIKEN (The Institute of Physical and Chemical Research), Wako, Saitama 351-0198, Japan \\ Tadatsugu Hatori \\ Department of Information Science, Kanagawa University, Hiratsuka, Kanagawa 256-1293, Japan \\ Tomokazu Kato \\ Department of Applied Physics, Waseda University, Shinjuku, Tokyo 169-8555, Japan
}

(Received 16 September 2002; accepted 13 May 2003)

\begin{abstract}
The diocotron instability in a low-density non-neutral electron plasma is examined via numerical simulations. For the simulations, a current-vortex filament model and a special-purpose computer, MDGRAPE-2 are used. In the previous work, a simulation method based on the current-vortex filament model, which is called "current-vortex method," is developed. It is assumed that electric current and vorticity have discontinuous filamentary distributions, and both point electric current and point vortex are confined in a filament, which is called "current-vortex filament." In this paper, the current-vortex method with no electric current is applied to simulations of the non-neutral electron plasma. This is equivalent to the traditional point-vortex method. MDGRAPE-2 was originally designed for molecular dynamics simulations. It accelerates calculations of the Coulomb interactions, the van der Waals interactions and so on. It can also be used to accelerate calculations of the Biot-Savart integral. The diocotron modes reproduced by the simulations agree with the result predicted by linear theory. This indicates that the current-vortex method is applicable to problems of the non-neutral plasma. The linear growth rates of the diocotron instability in the simulations also agree with the theoretical ones. This implies that MDGRAPE-2 gives the sufficiently accurate results for the calculations of the current-vortex method. A mechanism of merging of electron clumps is demonstrated by the simulations. It is concluded that the electric field induced by the conducting wall makes the nonlinear stage unstable and causes the clumps to merge. (C) 2003 American Institute of Physics. [DOI: 10.1063/1.1592516]
\end{abstract}

\section{INTRODUCTION}

Many features of non-neutral electron plasmas have been investigated both experimentally and theoretically. ${ }^{1-6}$ Above all, one of the most ubiquitous phenomena is the diocotron instability observed in a low-density $\left(\omega_{p e}^{2} \ll \omega_{c e}^{2}\right)$ nonneutral electron plasma column confined radially by a uniform axial magnetic field. The diocotron instability was first examined theoretically by MacFarlane et al. ${ }^{7}$ and Levy et al. ${ }^{8-10}$ and observed experimentally by Webster, ${ }^{11}$ Kapetanakos et al., ${ }^{12}$ and Peurrung et al. ${ }^{13}$ The linear theory for the diocotron instability has been developed and well understood. ${ }^{6,14}$ Thus we have chosen this phenomenon for the qualitative and quantitative benchmark of our simulation model (current-vortex filament model) and simulation method (a special-purpose computer, MDGRAPE-2).

As a simulation model, we use a current-vortex filament model. We have developed a two-dimensional "magnetohydrodynamic" vortex method. ${ }^{15}$ We call it the current-vortex method. The concept is based on the current-vortex filament

${ }^{a)}$ Electronic mail: yyanagi@phys.h.kyoto-u.ac.jp model. ${ }^{16-18}$ We assume that electric current and vorticity have discontinuous filamentary distributions. Both the point electric current and the point vortex are confined in each filament, which is called the current-vortex filament. By integrating the magnetic induction equation and the vorticity equation around a filament, we obtain time development equations of the current-vortex filaments. It is analytically shown that the two-dimensional equations of motion of lowdensity non-neutral electron plasmas with the guiding-center approximation coincide with those of the two-dimensional nonmagnetized fluids, i.e., the Euler equations. Thus, we apply the current-vortex method in the limit of no electric current to the two-dimensional electron plasmas. In this limit, the method is equivalent to the traditional point-vortex method. ${ }^{19-21}$

As a simulation method, we use a special-purpose computer, MDGRAPE-2. If no electric current is assumed, the current-vortex method needs the Biot-Savart integral to determine a flow velocity from (discretized) vorticities. Otherwise, the method needs the additional Biot-Savart integral to determine a magnetic field from electric currents. Unfortunately, it takes a considerable time to calculate the Biot- 
Savart integral in simulations. To improve performance of vortex simulations, one may needs special methods, such as the vortex-in-cell code. However, we took a way to use a special-purpose computer, MDGRAPE-2. It was originally designed for molecular dynamics simulations, and accelerates calculations of the Coulomb interactions, the van der Waals interactions, and so on. We find that MDGRAPE-2 can accelerate calculations of the Biot-Savart integral. You will see that MDGRAPE-2 is an "accelerator" not only for molecular dynamics simulations but also for plasma simulations.

The simulation results show that the model is valid for the electron plasmas, because the diocotron modes observed in the simulations are consistent with the theoretically predicted modes. The growth rates obtained by the simulations agree with the growth rates of linear theory. This indicates that MDGRAPE-2 gives sufficient precision for the simulations.

The angular velocities of the particles are influenced by the radius of the conducting wall. In the simulations of time evolution of two circular clumps, it is found that the direction of $\boldsymbol{E} \times \boldsymbol{B}$ force exerting the backward clump is outward, while the one exerting the forward clump is inward. The angular velocity of the backward clump, which is determined by the distance from the conducting wall, becomes larger than the forward one. Thus the backward clump catches up with the forward one from outside (near the wall) and merges with the forward one. It is concluded that the electric field induced by the conducting wall makes the nonlinear stage unstable and causes the clumps to merge.

In Sec. II, we present the simulation model, currentvortex filament model, the simulation method and the initial conditions. In Sec. III, we present simulation results. We compare the results to linear theory. The merging properties of the electron clumps produced by the diocotron instability are discussed. In Sec. IV, we give conclusions.

\section{SIMULATION MODEL AND METHOD}

In Sec. II A, the basic equations for non-neutral plasmas are given. In Sec. II B, the simulation model is discussed. The two-dimensional equations of motion of non-neutral plasmas with the guiding-center approximation coincide with the two-dimensional Euler equations. Thus we use the previously developed simulation model, current-vortex method, with no electric current. In this limit, our model reduces to the traditional point-vortex method. In Sec. II C, a specialpurpose computer, MDGRAPE-2, is briefly reviewed, which is used to accelerate the calculations of the Biot-Savart integral. In the last part of this section, we explain the initial conditions of the simulations.

\section{A. Basic equations}

We use the following basic equations:

$$
\begin{aligned}
& n_{e} m_{e}\left(\frac{\partial}{\partial t}+(\boldsymbol{u} \cdot \boldsymbol{\nabla})\right) \boldsymbol{u}=-\nabla p-e n_{e}(\boldsymbol{E}+\boldsymbol{u} \times \boldsymbol{B}), \\
& \frac{\partial n_{e}}{\partial t}+\boldsymbol{\nabla} \cdot\left(n_{e} \boldsymbol{u}\right)=0,
\end{aligned}
$$

$$
\begin{aligned}
& \boldsymbol{\nabla} \cdot \boldsymbol{E}=\frac{e n_{e}}{\epsilon_{0}}, \\
& \omega_{z}=\hat{z} \cdot \nabla \times \boldsymbol{u}, \\
& \boldsymbol{B}=B_{0} \hat{z},
\end{aligned}
$$

where $n_{e}, m_{e}, e, p$, and $B_{0}$ are the number density of electrons, the electron mass, the electron charge, the kinetic pressure, and the uniform magnetic field in the $z$ direction, respectively. Notation $\omega_{z}$ is the z-component of the vorticity. Notations $\boldsymbol{u}, \boldsymbol{B}$, and $\boldsymbol{E}$ are the flow velocity, the magnetic field, and the electric field on the $x-y$ plane. A unit vector in $z$ direction is denoted by $\hat{z}$. Equations (1), (2), and (3) are the equation of motion, the equation of continuity, and Gauss' theorem, respectively.

In the present analysis, a cold-fluid guiding-center model is adopted in which electron inertial effects are neglected. The motion of a strongly magnetized electron fluid element is determined from

$$
e n_{e}(\boldsymbol{E}+\boldsymbol{u} \times \boldsymbol{B})=0 .
$$

In the electrostatic approximation, the electric field is determined by the scalar potential $\phi$, namely, $\boldsymbol{E}=-\boldsymbol{\nabla} \phi$, and Eq. (6) gives

$$
\begin{aligned}
& \boldsymbol{u}=-\frac{1}{B_{0}} \boldsymbol{\nabla} \phi \times \hat{z}, \\
& \boldsymbol{\nabla} \cdot \boldsymbol{u}=0 .
\end{aligned}
$$

By means of Eq. (4), the vorticity $\omega_{z}$ reduces to

$$
\omega_{z}=\frac{e n_{e}}{\epsilon_{0} B_{0}}=\frac{\omega_{p e}^{2}}{\omega_{c e}}=2 \omega_{D},
$$

where $\omega_{p e}=\left(n_{e} e^{2} /\left(\epsilon_{0} m_{e}\right)\right)^{1 / 2}$ and $\omega_{c e}=e B_{0} / m_{e}$. Notation $\omega_{D}$ is the diocotron frequency. Equation (9) means that the vorticity is proportional to the electron density $n_{e}$ or the diocotron frequency $\omega_{D}$.

On the other hand, two-dimensional flow velocity is determined by the Euler equation. Namely, the flow velocity is obtained by the stream function $\psi$,

$$
\boldsymbol{u}=-\boldsymbol{\nabla} \psi \times \hat{z} .
$$

It follows from Eq. (7) that the scalar potential $\phi$ is related to the stream function, $\psi=\phi / B_{0} \cdot{ }^{8,10}$ The electron fluid motion, therefore, can be determined by the two-dimensional Euler equations.

\section{B. Simulation model}

Here we explain the current-vortex method used in the simulations. We use the two-dimensional ideal magnetohydrodynamic equations,

$$
\begin{aligned}
& \frac{\partial \omega_{z}}{\partial t}=-(\boldsymbol{u} \cdot \boldsymbol{\nabla}) \omega_{z}+(\boldsymbol{B} \cdot \boldsymbol{\nabla}) j_{z}, \\
& \frac{\partial A_{z}}{\partial t}=-(\boldsymbol{u} \cdot \boldsymbol{\nabla}) A_{z}, \\
& \boldsymbol{\nabla} \cdot \boldsymbol{u}=0,
\end{aligned}
$$




$$
\begin{aligned}
& \boldsymbol{E}+\boldsymbol{u} \times \boldsymbol{B}=0, \\
& \boldsymbol{B}=-\hat{z} \times \nabla A_{z}, \\
& \omega_{z}=\hat{z} \cdot \nabla \times \boldsymbol{u}, \\
& j_{z}=\frac{\hat{z}}{\mu_{0}} \cdot \nabla \times \boldsymbol{B},
\end{aligned}
$$

where $A_{z}$ and $j_{z}$ are the $z$-components of the magnetic vector potential and the electric current density, respectively. The mass density is normalized to unity.

We assume that the electric current and the vorticity have discontinuous filamentary distributions, and the point electric current and the point vortex are confined in each filament coaxially. In other words, the electric current and the vorticity always align and form the current-vortex filaments. It follows that the electric current density $j_{z}(\boldsymbol{r}, t)$ and the vorticity $\omega_{z}(\boldsymbol{r}, t)$ are denoted by

$$
\begin{aligned}
& j_{z}(\boldsymbol{r}, t)=\sum_{i} J_{i}(t) \delta\left(\boldsymbol{r}-\boldsymbol{r}_{i}(t)\right), \\
& \omega_{z}(\boldsymbol{r}, t)=\sum_{i} \Omega_{i}(t) \delta\left(\boldsymbol{r}-\boldsymbol{r}_{i}(t)\right),
\end{aligned}
$$

where $\delta(\boldsymbol{r})$ is Dirac's two-dimensional delta function. The notation $\boldsymbol{r}_{i}(t)$ is the position vector of the $i$ th current-vortex filament, $J_{i}(t)$ and $\Omega_{i}(t)$ are the total electric current and the circulation inside the $i$ th filament. Equations (18) and (19) directly show that there is the current-vortex filament at $\boldsymbol{r}_{i}(t)$.

We rewrite the vorticity Eq. (11) and the magnetic induction Eq. (12) in terms of the filamentary representations (18) and (19). By integrating the resulting equations over a circle area whose center and radius are $\boldsymbol{r}_{k}$ and $\epsilon$, respectively, we obtain solutions concerning the specific filament, say the $k$ th filament. The solutions are given by

$$
\begin{aligned}
& \frac{d \boldsymbol{r}_{k}}{d t}=\boldsymbol{u}\left(\boldsymbol{r}_{k}, t\right)-\frac{J_{k}(t)}{\Omega_{k}(t)} \boldsymbol{B}\left(\boldsymbol{r}_{k}, t\right), \\
& \frac{d \Omega_{k}(t)}{d t}=0, \\
& \frac{d J_{k}(t)}{d t}=0,
\end{aligned}
$$

where

$$
\begin{aligned}
& \boldsymbol{u}\left(\boldsymbol{r}_{k}, t\right)=\sum_{i \neq k} \Omega_{i}(t) \nabla G\left(\boldsymbol{r}_{k}-\boldsymbol{r}_{i}\right) \times \hat{z}, \\
& \boldsymbol{B}\left(\boldsymbol{r}_{k}, t\right)=\sum_{i \neq k} J_{i}(t) \boldsymbol{\nabla} G\left(\boldsymbol{r}_{k}-\boldsymbol{r}_{i}\right) \times \hat{z},
\end{aligned}
$$

and $G(\boldsymbol{r})$ is the two-dimensional Green function that obeys

$$
\boldsymbol{\nabla}^{2} G(\boldsymbol{r})=-\delta(\boldsymbol{r}) .
$$

Equations (20), (21), and (22) give the equation of motion of the $k$ th filament, the conservation laws of the circulation and the total electric current inside the $k$ th filament, respectively.

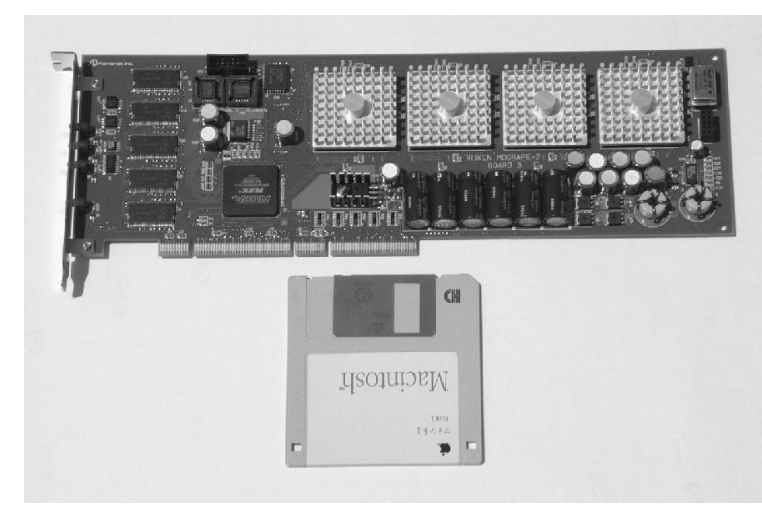

FIG. 1. A photograph of MDGRAPE-2 is shown.

The equations determine the time evolutions of the currentvortex filaments. In the limit of $J_{i}(t)=0$, Eqs. (20)-(24) reduce to the following normalized equations:

$$
\begin{aligned}
& \frac{d \boldsymbol{r}_{k}}{d t}=\boldsymbol{u}\left(\boldsymbol{r}_{k}, t\right), \\
& \frac{d \Omega_{k}(t)}{d t}=0, \\
& \boldsymbol{u}\left(\boldsymbol{r}_{k}, t\right)=\sum_{i \neq k} 2 \pi \Omega_{i}(t) \nabla G\left(\boldsymbol{r}_{k}-\boldsymbol{r}_{i}\right) \times \hat{z} .
\end{aligned}
$$

The factor $2 \pi$ in the Biot-Savart integral (28) is introduced to cancel out the factor $1 /(2 \pi)$ in the Green function. We use these normalized equations in the simulations, because the equations to determine the time evolution of twodimensional non-neutral electron plasmas with guidingcenter approximation have the same form as the twodimensional Euler equations for nonmagnetized fluids. To incorporate the effect of the conducting wall around the electrons, we use the method of images. ${ }^{22}$

\section{Simulation method}

Equation (28) is the Biot-Savart integral. One must notice that it takes a considerable time to calculate the BiotSavart integral in simulations. Usually the calculation time is proportional to $N^{2}$, where $N$ is the number of mesh points. In this work, we use a special-purpose computer, MDGRAPE-2, to accelerate the calculations of the BiotSavart integral. A photograph of MDGRAPE-2 is shown in Fig. 1. MDGRAPE-2 is a standard PCI board that can be installed even on Pentium III based PCs. It was originally designed for molecular dynamics simulations, and accelerates the calculations of the Coulomb interactions, the van der Waals interactions, and so on. ${ }^{23}$ The calculation speed of MDGRAPE-2 depends on the number of particles that interact with each other and the number of boards installed on a PC. This is due to the communication time between the host CPU and PCI board. If the number of particles exceeds $10^{5}$, the communication time is not negligible. In such a case, it is possible to implement the well known fast algorithms, such as the vortex-in-cell method and the pseudoparticle multipole method, on MDGRAPE-2 to reduce the communication 


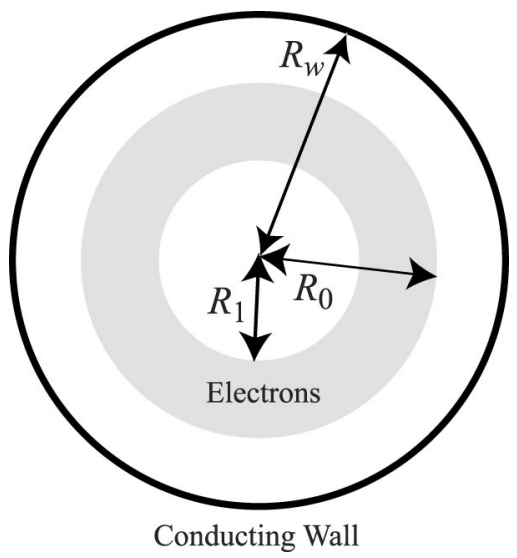

FIG. 2. An initial condition of the simulations is shown. A perfectly conducting wall is located at $R_{w}$. Outer and inner radii of the distribution of electrons are denoted by $R_{0}$ and $R_{1}$, respectively.

time. ${ }^{21,24}$ For our case, however, the maximum number of the particles is $10^{4}$ and there is not enough merit to implement the fast algorithm. Thus we simply use MDGRAPE-2 to accelerate the calculation of Biot-Savart integral in the traditional point-vortex method. In this case, the maximum calculation speed still reaches 120 GFlops, if the number of particles is $10^{5}$ and the number of MDGRAPE- 2 boards is 4 . It is about 8 times faster than the fastest vector-type supercomputer with one processor. The method how to calculate the Biot-Savart integral on MDGRAPE-2 is given in Ref. 15.

\section{Initial conditions}

An initial configuration of the electrons is shown in Fig. 2. A perfectly conducting wall is located at $R_{w}$. Outer and inner radii of the distribution of electrons are denoted by $R_{0}$ and $R_{1}$, respectively. The electron distribution is represented by the sum of point vortices in the simulations. The maximum number of point vortices is $10^{4}$. Each point vortex has circulation $0.15 \Gamma_{s}$. The outer radius of the electron distribution is $R_{0}=50 L_{s}$. Here the notations $\Gamma_{s}$ and $L_{s}$ are introduced for normalization. The average vorticity $\bar{\omega}_{z}$ is obtained by

(a)
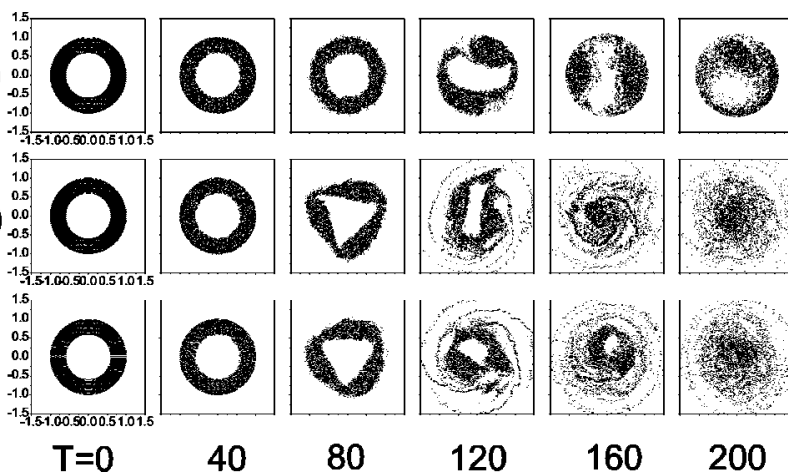

(b)
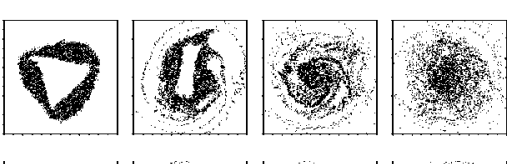

(c)
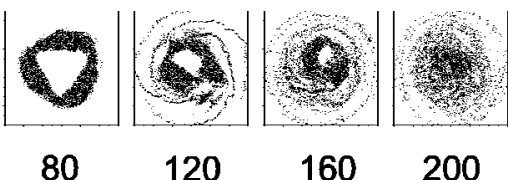

FIG. 3. Time evolutions of the electron distributions at $T=0,40,80,120$, 160 , and 200 are shown. Initial inner radius of the distribution of electrons is $R_{1}=0.6 R_{0}$. Conducting wall is located at $R_{w}=$ (a) $1.1 R_{0}$, (b) $1.6 R_{0}$, and (c) $\infty$, respectively.

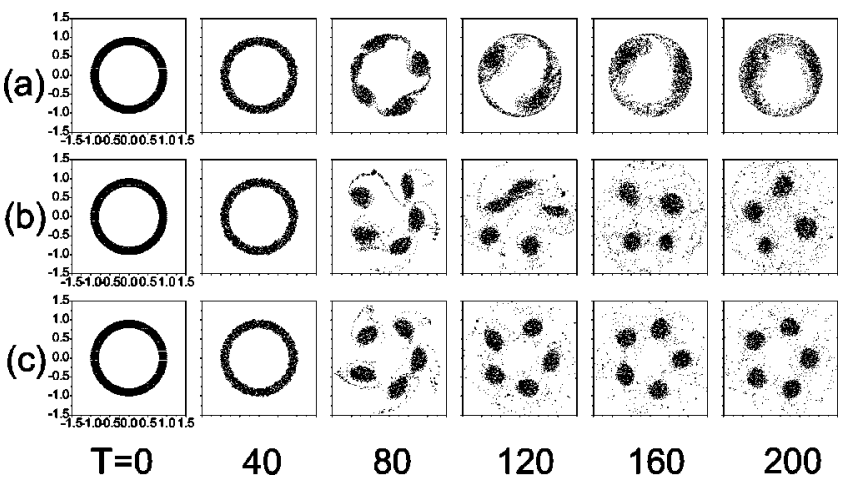

FIG. 4. Time evolutions of the electron distributions at $T=0,40,80,120$, 160 , and 200 are shown. Initial inner radius of the distribution of electrons is $R_{1}=0.8 R_{0}$. Conducting wall is located at $R_{w}=$ (a) $1.1 R_{0}$, (b) $1.6 R_{0}$, and (c) $\infty$, respectively.

$$
\bar{\omega}_{z}=\frac{0.15 \Gamma_{s} \times 10^{4}}{\pi\left(50 L_{s}\right)^{2}}=1.91 \times 10^{-1} \frac{\Gamma_{s}}{L_{s}^{2}} .
$$

In this case, the diocotron frequency $\omega_{D}=\omega_{p e}^{2} /\left(2 \omega_{c e}\right)$ is determined by the average vorticity as $\omega_{D}=\bar{\omega}_{z} / 2$. The value of the diocotron frequency becomes

$$
\omega_{D}=\frac{e n_{e}}{2 \epsilon_{0} B_{0}}=\frac{1}{2} \bar{\omega}_{z}=9.55 \times 10^{-2} \frac{\Gamma_{s}}{L_{s}^{2}} .
$$

Thus the vorticity is proportional to the electron number density. In the simulations, we set $T=L_{s}^{2} / \Gamma_{s}=1$ for normalization, where $T$ means the time in the simulation. Time step is $\Delta T=10^{-4}$. All the length scales in the simulations are normalized by $R_{0}$ from now on.

\section{SIMULATION RESULTS OF DIOCOTRON INSTABILITY}

\section{A. Time evolution of annular electron distribution}

In Figs. 3-5 time evolutions of the electron distributions at $T=0,40,80,120,160$, and 200 are shown. There are two main parameters in these simulations. One is a ratio of inner to outer radii of the electron distribution $R_{1} / R_{0}$, which is chosen as 0.6 in Fig. 3, 0.8 in Fig. 4, and 0.9 in Fig. 5. The other is a radius of the conducting wall $R_{w}$, which is chosen as $1.1 R_{0}$ in (a), $1.6 R_{0}$ in (b), and $\infty$ in (c) in Figs. 3 and 4 , and $\infty$ in Fig. 5.

The simulation results show that the linearly most unstable modes are 2 at $T=120$ in (a), 3 at $T=80$ in (b), 3 at $T=80$ in (c) in Fig. 3, 4 at $T=80$ in (a), 5 at $T=80$ in (b), 5 at $T=80$ in (c) in Fig. 4, and 9 at $T=80$ in Fig. 5. The unstable modes obtained by the simulations agree with the

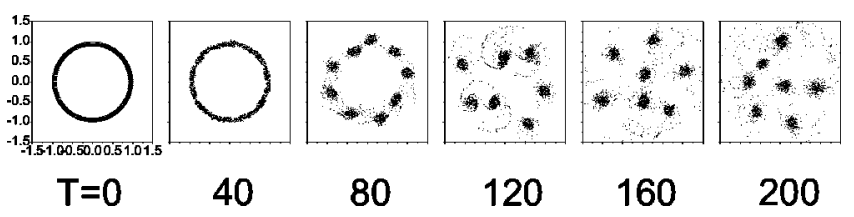

FIG. 5. Time evolution of the electron distribution at $T=0,40,80,120$, 160 , and 200 is shown. Initial inner radius of the distribution of electrons is $R_{1}=0.9 R_{0}$. Conducting wall is located at $R_{w}=\infty$. 
TABLE I. Unstable modes obtained by the linear theory in descending order of the growth rate are shown.

\begin{tabular}{ccc}
\hline \hline$R_{1} / R_{0}$ & $R_{w}$ & Unstable modes \\
\hline 0.6 & 1.1 & 2 \\
0.6 & 1.6 & 3,2 \\
0.6 & $\infty$ & 3 \\
0.8 & 1.1 & $4,3,5$ \\
0.8 & 1.6 & $5,4,6$ \\
0.8 & $\infty$ & $5,4,6$ \\
0.9 & $\infty$ & $9,8,10$ \\
\hline
\end{tabular}

theoretical results shown in Table I. It indicates that the current-vortex method is qualitatively valid for problems of the non-neutral electron plasmas.

It is interesting that the final stage after the linear growth stage strongly depends on the radius of the conducting wall $R_{w}$. In Fig. 3, mode 1 appears in (a), while the electron distributions exhibit center-peaked broad profiles in (b) and (c). In Fig. 4 also, the modes observed in the final stages are 2,4 , and 5 , respectively. These results indicate that the conducting wall strongly affects the electron motions inside it. We discuss the merging properties of the electron clumps in Sec. III C.

\section{B. Growth rate}

To check if the simulation results are quantitatively valid, we compare the growth rates of the diocotron instability obtained by the simulations to the analytical ones.

The growth rate of the diocotron instability has been reported by Davidson et al. ${ }^{6,14}$ They assume the annular electron distribution, which is the same condition as the one we use in the simulations. A dispersion relation for complex eigenfrequency $\omega$ is given by

$$
\left(\frac{\omega}{\omega_{D}}\right)^{2}-b_{\ell} \frac{\omega}{\omega_{D}}+c_{\ell}=0,
$$

where $\omega_{D}=\omega_{p e}^{2} / 2 \omega_{c e}=n_{e} e /\left(2 \epsilon_{0} B\right)$ and

$$
b_{\ell}=\ell\left[1-\left(\frac{R_{1}}{R_{0}}\right)^{2}\right]+\left[1-\left(\frac{R_{1}}{R_{0}}\right)^{2 \ell}\right]\left(\frac{R_{0}}{R_{w}}\right)^{2 \ell},
$$

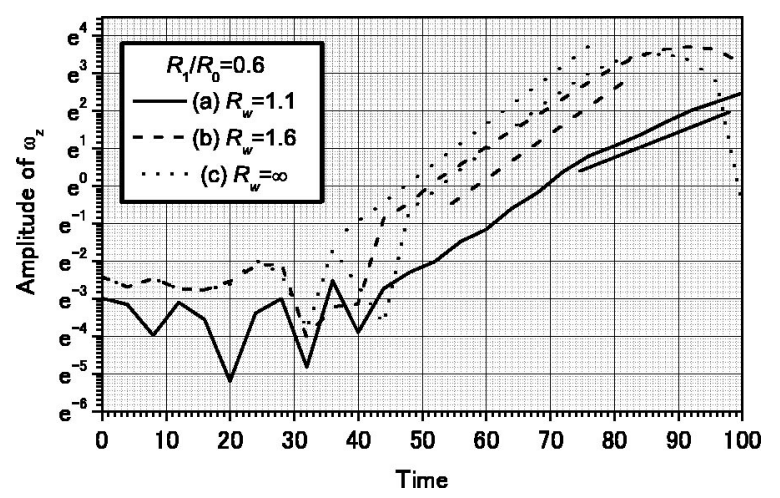

FIG. 6. Time evolutions of the linearly most unstable Fourier coefficients are shown that correspond to Fig. 3. The azimuthal mode numbers are 2 in (a) and 3 in (b) and (c).

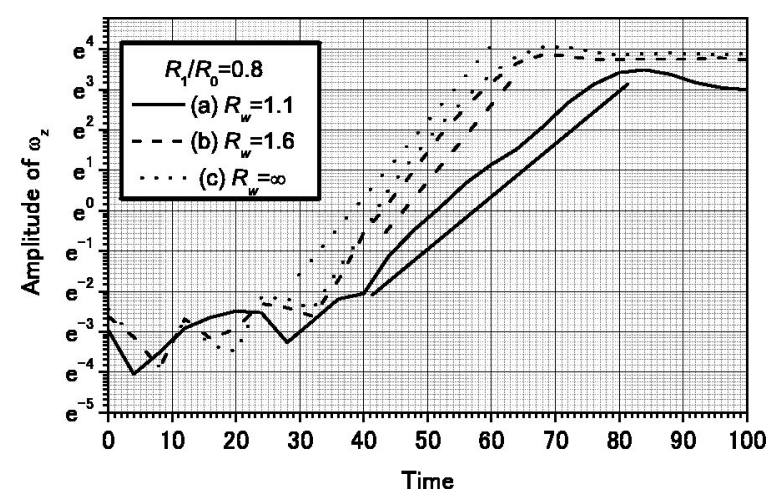

FIG. 7. Time evolutions of the linearly most unstable Fourier coefficients are shown that correspond to Fig. 4. The azimuthal mode numbers are 4 in (a) and 5 in (b) and (c).

$$
\begin{aligned}
c_{\ell}= & \ell\left[1-\left(\frac{R_{1}}{R_{0}}\right)^{2}\right]\left[1-\left(\frac{R_{1}}{R_{w}}\right)^{2 \ell}\right]-\left[1-\left(\frac{R_{0}}{R_{w}}\right)^{2 \ell}\right] \\
& \times\left[1-\left(\frac{R_{1}}{R_{0}}\right)^{2 \ell}\right] .
\end{aligned}
$$

For our case, there is no central conductor. Thus the terms that arise from the charges on the central conductor are omitted in Eqs. (32) and (33). The solutions of Eq. (31) are

$$
\omega=\frac{1}{2} \omega_{D}\left(b_{\ell} \pm \sqrt{b_{\ell}^{2}-4 c_{\ell}}\right) .
$$

If $4 c_{\ell}>b_{\ell}^{2}$, the solutions are complex, which correspond to instability. Using Eq. (34), detailed instability properties can be investigated for the specific electron distribution.

Time evolutions of the Fourier coefficients of $\omega_{z}$ for Fig. 3 is shown in Fig. 6, for Fig. 4 in Fig. 7 and for Fig. 5 in Fig. 8 , respectively. The curved lines indicate the values of the coefficients obtained by the simulations, and straight lines indicate the growth rates obtained by linear theory. We can see that the slopes of the curves during the linear growth stage are approximately the same as those of the straight lines. The exact values are plotted in Fig. 9. The growth rates obtained by the simulations agree with the growth rates of linear theory. This indicates that MDGRAPE-2 gives the sufficient precision for the simulations.

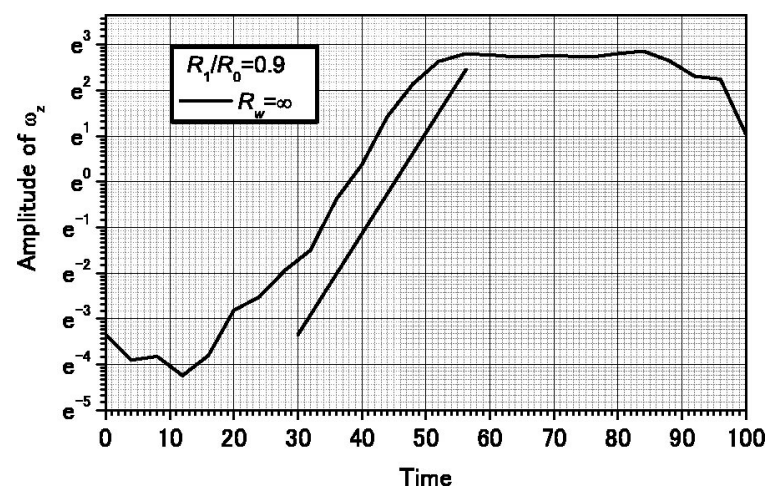

FIG. 8. Time evolution of the linearly most unstable Fourier coefficient is shown that corresponds to Fig. 5. The azimuthal mode number is 9 . 


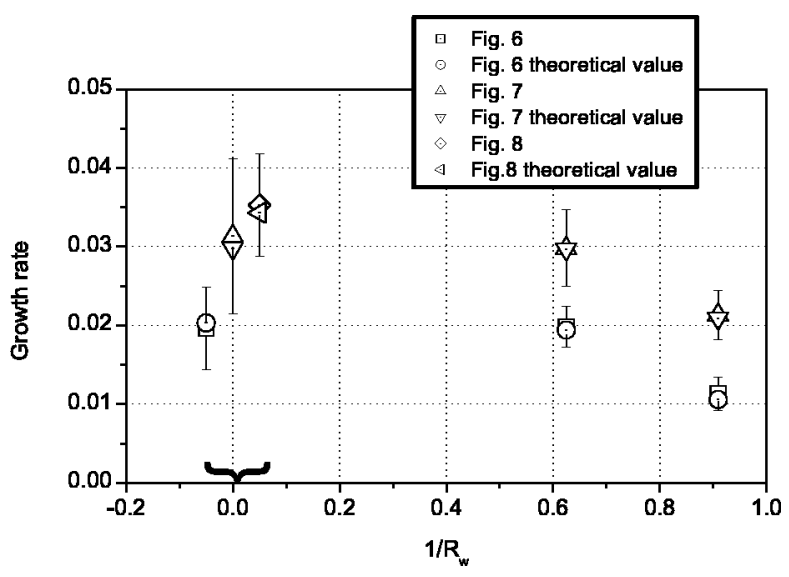

FIG. 9. Both growth rates obtained by the simulations and linear theory are plotted. The leftmost three data correspond to the growth rates at $1 / R_{w}=0$. To prevent the data from overlapping with each other, we plot them in the different positions.

\section{Boundary effect}

Here we discuss the boundary effect on the electron clumps. We focus on the results shown in Fig. 4. The figure shows that the merging of the clumps is strongly influenced by the radius of the conducting wall. More mergers occur if the wall radius is smaller. To see the merging properties quantitatively, we plot time evolutions of angular positions of particles in Fig. 10.

In Fig. 10(a), initial particles are selected from the electron distribution in Fig. 4(a), $T=0$ at $\theta=n \pi / 10, r=0.93 R_{0}$ where $n=0,1, \ldots, 19$. In the same manner, initial particles in Figs. 10(b) and 10(c) are selected from Figs. 4(b) and 4(c), respectively. A bundle of lines indicates a clump. Note that the ordinate has the period of $2 \pi$. Slope of a line indicates angular velocity of a particle. Durations of the linear growth stages are obtained from Fig. 7. They are $T=40-80$ in (a) and $T=35-60$ in (b) and (c). In these durations, the slopes of the lines in Fig. 9, which are initially the same, gradually change, depending on the wall radius. Thus it is found that the transition from the linear growth stage to the nonlinear stage yields the change of the slopes. In Figs. 10(b) and $10(\mathrm{c})$, the slopes of the lines are approximately constant during the nonlinear stage. It indicates that the nonlinear stage is

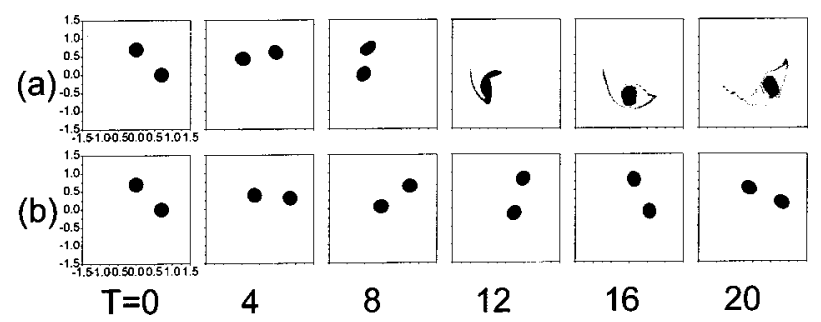

FIG. 11. Time evolutions of two circular clumps are plotted. The two clumps are located at $(x, y)=\left(0.7 R_{0}, 0\right)$ and $\left(0,0.7 R_{0}\right)$. The initial radii of the clumps are $0.2 R_{0}$ each. The conducting walls are located at $r=R_{0}$ in (a) and $r=\infty$ in (b).

stable and clumps rotate in the nearly constant angular velocity. On the other hand, the slopes of the lines in Fig. 10(a) are still varying in the nonlinear stage, that indicates the nonlinear stage is unstable. This is due to the smaller wall radius compared with those in Figs. 10(b) and 10(c). Although the details are omitted here, we have carried out the other simulations, where we use the electron distributions at $T=160$ in Figs. 4(b) and 4(c) with the conducting wall relocated at $R_{w}=R_{0}$ as initial conditions. In these simulations, the clumps no longer keep their initial shapes and merge with each other. The final distributions become broad ones. Thus we conclude that the electric field induced by the conducting wall makes the nonlinear stage unstable and causes the clumps to merge.

Next, we consider a mechanism by which two clumps merge. In Fig. 11, we show time evolution of two circular clumps. The number of particles in each clump is 1000, which is the approximate number of particles in each clump in Fig. 4(c) at $T=200$. The centers of the two clumps are initially located at $(x, y)=\left(0.7 R_{0}, 0\right)$ and $\left(0,0.7 R_{0}\right)$. The initial radii of the clumps are $0.2 R_{0}$ each. The conducting walls are located at $r=R_{0}$ in (a) and $r=\infty$ in (b). Note that the time interval between the snapshots is 4 in Fig. 11, while the ones of the other figures are 40. In Fig. 11(a), we can see that the two clumps merge rapidly near the wall. As is shown in Fig. 11(b), the circular two clumps without the conducting wall move like binary stars if the motion of the clumps is restricted in a two-dimensional plane. In addition, we have checked by the simulations that only one clump surrounded by the conducting wall survives stably. Its shape remains (a)

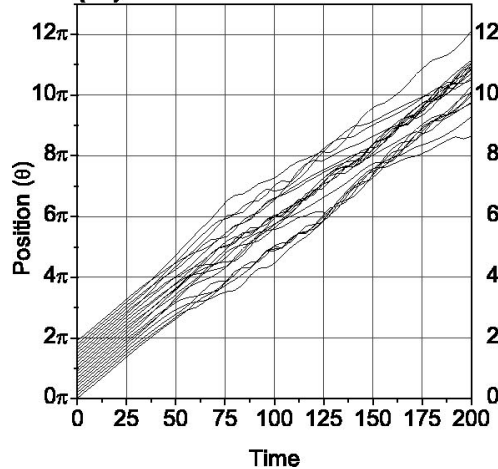

(b)

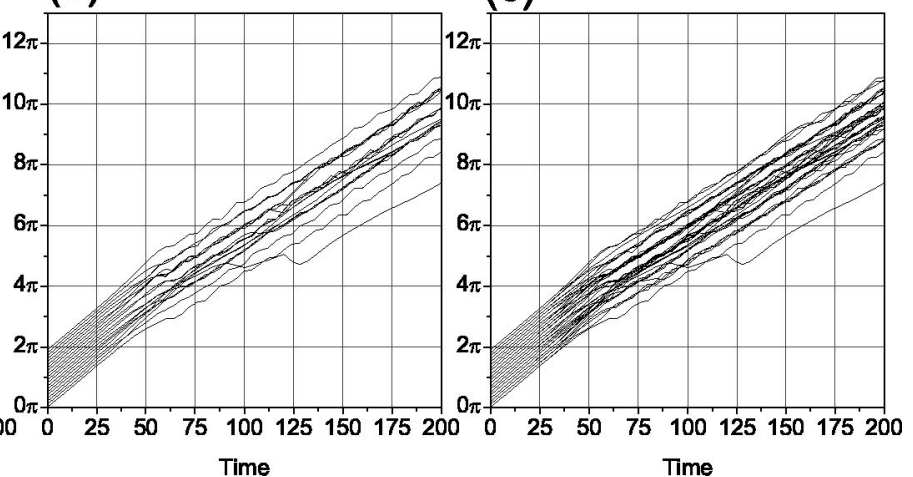

FIG. 10. Time evolutions of angular positions of particles are plotted. The initial positions of the traced particles in (a), (b), and (c) are at $\theta=n \pi / 10, T$ $=0,(n=0,1,2, \ldots, 19)$ in Figs. 4(a), 4(b), and 4(c), respectively. 
almost circular. In this case, however, the two clumps merge by the electric field induced by the conducting wall. Thus we conclude that the merging of the two clumps are driven by the electric field induced by each other. The direction of the $\boldsymbol{E} \times \boldsymbol{B}$ force exerted on the forward clump in the counterclockwise rotation is inward, while the force on the backward clump is outward. Here we use the terms "forward" and "backward" in regard to the direction of orbital rotation. The angular velocity of the backward clump, which is determined by the radial electric field that depends on the distance from the conducting wall, is larger than that of the forward clump, because the distance from the backward clump to the conducting wall is smaller than that from the forward clump. Thus the backward clump catches up with the forward one from outside (near the wall) and merges with the forward one.

\section{CONCLUSIONS}

In this paper we have shown the simulation results of non-neutral electron plasmas. The simulation model we use is the current-vortex method. In the simulations we use MDGRAPE-2 to accelerate calculations of the Biot-Savart integral.

In the limit of no electric current, the current-vortex method coincides with the traditional point-vortex method, and is qualitatively valid for the non-neutral plasma simulations, because the most unstable modes are reproduced correctly by the simulations. The growth rates observed in the simulations also agree with the theoretical ones, which indicates that MDGRAPE-2 gives sufficient precision for the simulations.

It is concluded that the electric field induced by the conducting wall makes the nonlinear stage unstable and causes the clumps to merge. In the simulations of time evolution of two circular clumps, it is found that the direction of $\boldsymbol{E} \times \boldsymbol{B}$ force exerting the backward clump is outward, while the one exerting the forward clump is inward. The angular velocity of the backward clump, which is determined by the distance from the conducting wall, becomes larger than the forward one. Thus the backward clump catches up with the forward one from outside (near the wall) and merges with the forward one.

In the present analysis of the merging properties, we limit ourselves to the two-clump system. However, the merging properties of $N$-clump system is still unclear. The stability analysis of vortex arrays has been presented by Campbell. ${ }^{25}$ The result may give us a clue to study the nonlinear behavior of the electron clumps.

\section{ACKNOWLEDGMENT}

This work was supported by the Special Postdoctoral Research Program at RIKEN.

\section{APPENDIX: INITIAL PERTURBATIONS INCLUDED IN INITIAL CONDITIONS OF SIMULATIONS}

First, we show two simulation results in Fig. 12. The values of $R_{w}$ and $R_{1}$ in (a) are $\infty$ and $0.8 R_{0}$, respectively, (a)
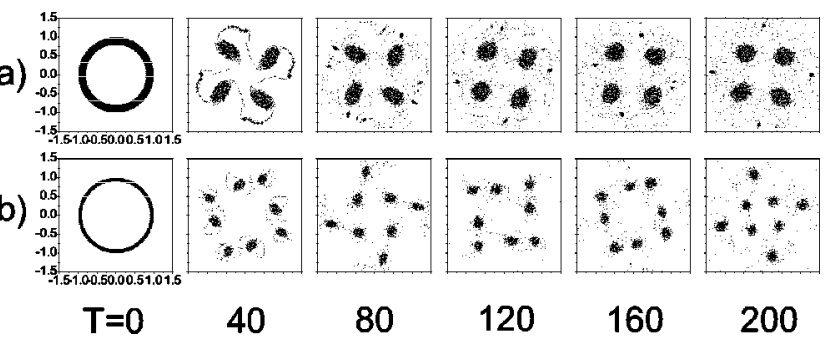

FIG. 12. Time evolutions of the electron distributions at $T=0,40,80,120$, 160 , and 200 are shown. Initial inner radii of the distributions of the electrons are $R_{1}=$ (a) $0.8 R_{0}$ and (b) $0.9 R_{0}$, respectively. Initial arrangements of the particles used here are shown in Fig. 13(a).

which are the same as Fig. 4(c). Those in (b) are $\infty$ and $0.9 R_{0}$, respectively, which are the same as Fig. 5 . Note that the linearly most unstable mode is 5 in Fig. 12(a) and 9 in Fig. 12(b), while the obtained mode is 4 and 8 , respectively. This is due to the initial perturbations. The particles inside the annular electron distributions in Figs. 3-5 are arranged as in Fig. 13(b). On the other hand, the particles in Fig. 12
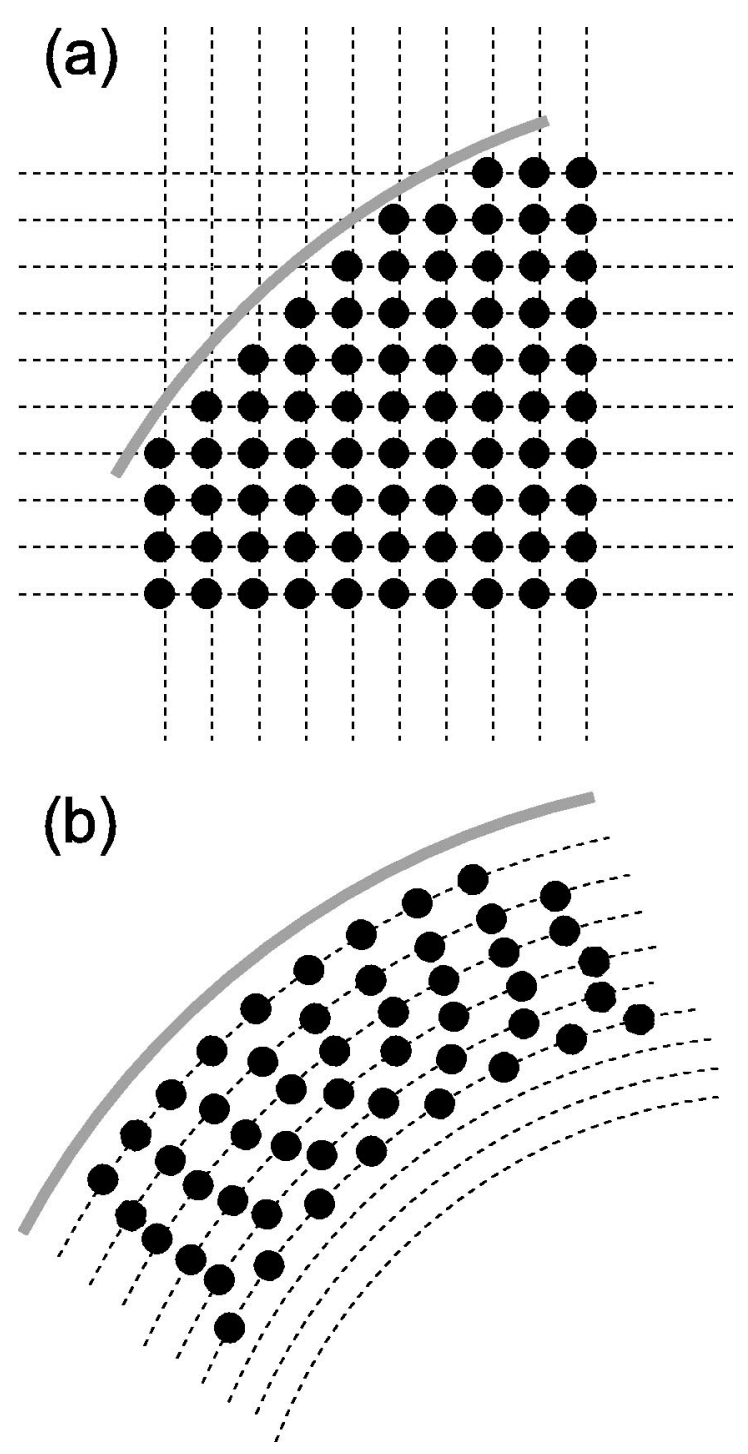

FIG. 13. Initial arrangements of the particles are shown. 


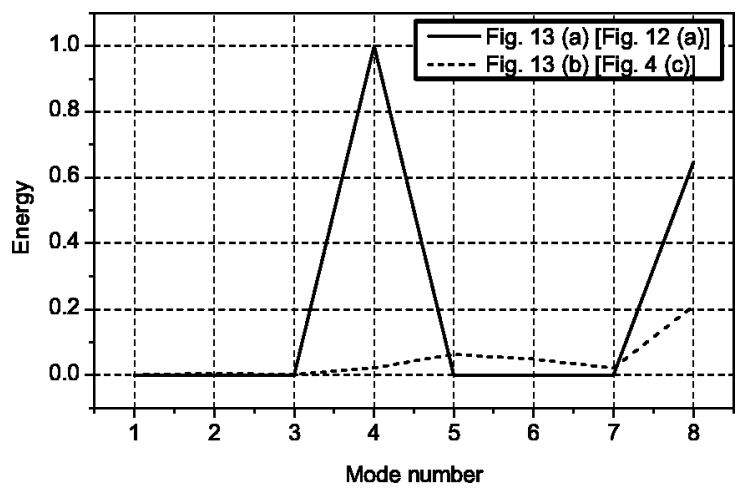

FIG. 14. The values of the initial energy in several modes are plotted.

are arranged as in Fig. 13(a). For the cases shown in Fig. 12, mode 4 and its higher harmonic modes gain more initial energy than the other modes, such as 3,5 , and so on. The values of the initial energy in some mode numbers are shown in Fig. 14. In this figure, the values are normalized by the initial energy of mode 4 in the case of Fig. 13(a). It is found that the initial energy of modes 4 and 8 in the case of Fig. 13(a) is much larger than the other ones. Thus, modes 4 and 8 dominate because they are initialized with much greater amplitude. We conclude that the unstable modes are very sensitive to the initial energy included in the initial condition.
${ }^{1}$ Y. Kiwamoto, K. Ito, A. Sanpei, and A. Mohri, Phys. Rev. Lett. 85, 3173 (2000).

${ }^{2}$ Y. Kiwamoto, K. Ito, A. Sanpei, A. Mohri, T. Yuyama, and T. Michishita, J. Phys. Soc. Jpn. 68, 3766 (1999).

${ }^{3}$ D. A. Schecter, D. H. E. Dubin, K. S. Fine, and C. F. Driscoll, Phys. Fluids 11, 905 (1999).

${ }^{4}$ D. A. Schecter, D. H. E. Dubin, A. C. Cass, C. F. Driscoll, I. M. Lansky, and T. M. O'Neil, Phys. Fluids 12, 2397 (2000).

${ }^{5}$ D. A. Schecter and D. H. E. Dubin, Phys. Fluids 13, 1704 (2001).

${ }^{6}$ R. C. Davidson, An Introduction to the Physics of Non-Neutral Plasmas (Addison-Wesley, Palo Alto, CA, 1990), Chap. 6.

${ }^{7}$ C. C. MacFarlane and H. G. Hay, Proc. Phys. Soc. London, Sect. B 63, 409 (1950).

${ }^{8}$ R. H. Levy, Phys. Fluids 8, 1288 (1965).

${ }^{9}$ R. H. Levy, Phys. Fluids 11, 920 (1968).

${ }^{10}$ R. H. Levy, J. D. Daugherty, and O. Buneman, Phys. Fluids 12, 2616 (1969).

${ }^{11}$ H. F. Webster, J. Appl. Phys. 26, 1386 (1955).

${ }^{12}$ C. A. Kapetanakos, D. A. Hammer, C. Striffler, and R. C. Davidson, Phys. Rev. Lett. 30, 1303 (1973).

${ }^{13}$ A. J. Peurrung and J. Fajans, Phys. Fluids A 5, 493 (1993).

${ }^{14}$ R. C. Davidson and G. M. Felice, Phys. Plasmas 5, 3497 (1998).

${ }^{15}$ Y. Yatsuyanagi, T. Ebisuzaki, T. Hatori, and T. Kato, Phys. Plasmas 10, 3181 (2003).

${ }^{16}$ Y. Yatsuyanagi, T. Hatori, and T. Kato, J. Phys. Soc. Jpn. 65, 745 (1996).

${ }^{17}$ Y. Yatsuyanagi, T. Hatori, and T. Kato, J. Phys. Soc. Jpn. 67, 166 (1998).

${ }^{18}$ Y. Yatsuyanagi, T. Hatori, and T. Kato, J. Plasma Phys. 62, 493 (1999).

${ }^{19}$ J. P. Christiansen, J. Comput. Phys. 135, 189 (1997).

${ }^{20}$ P. Koumoutsakos, J. Comput. Phys. 138, 821 (1997).

${ }^{21}$ A. Leonard, J. Comput. Phys. 37, 289 (1980).

${ }^{22}$ J. D. Jackson, Classical Electrodynamics (Wiley, New York, 1975), Chap. 2.

${ }^{23}$ T. Narumi, R. Susukita, T. Ebisuzaki, G. McNiven, and B. Elmegreen, Mol. Simul. 21, 401 (1999).

${ }^{24}$ A. Kawai and J. Makino, Astrophys. J. Lett. 550, L143 (2001).

${ }^{25}$ L. J. Campbell, Phys. Rev. A 24, 514 (1981). 